\title{
Comparison of the Postoperative Analgesic Effects of Levobupivacaine Wound Instillation with a Placebo after Unilateral Inguinal Hernia Repair: A Randomized- controlled Trial
}

\author{
Maja Šujica ${ }^{1}$, Tatjana Gazibara ${ }^{2}$, Ana Mandraš $^{1}$, Igor Krunićn ${ }^{1}$, Sonja Vučković ${ }^{3}$ \\ ${ }_{1}$ "Dr Vukan Čupić" Mother and Child Health Institute, Belgrade, Serbia, ${ }^{2}$ Institute of Epidemiology, Faculty of Medicine, \\ University of Belgrade, Belgrade, Serbia, ${ }^{3}$ Department of Pharmacology, Clinical Pharmacology and Toxicology, Faculty of \\ Medicine, University of Belgrade, Belgrade, Serbia
}

Correspondence: sujicamaja@gmail.com; Tel.: + 381641708 294; Fax.: + 381112697232

Received: December 9, 2020; Accepted: September 5, 2021

\begin{abstract}
Objective - To compare the effectiveness and safety of wound instillation with levobupivacaine with a placebo, in children who underwent inguinal hernia repair, for pain relief. The secondary objective was to examine the frequency of postoperative analgesic mixture (paracetamol/ibuprofen) use. Methods - Single center randomized placebo-controlled trial. Paediatric teaching hospital in Belgrade, Serbia. This study included 100 children who underwent elective surgery for unilateral inguinal hernia. Children were randomized by simple randomization into two groups ( $\mathrm{N}=50$ in the experimental group, $\mathrm{N}=50$ in the control group). A solution of $0.5 \%$ levobupivacaine, $0.5 \mathrm{mg} / \mathrm{kg}(0.1 \mathrm{ml} / \mathrm{kg})$ was instilled into the wounds of children in the experimental group before suturing the abdominal fascia. The same amount of $0.9 \%$ saline was administered to the children in the control group. The primary outcome was the level of postoperative pain after coming round from general anesthesia $(\mathrm{t} 0)$ and at $2 \mathrm{~h}, 6 \mathrm{~h}, 12 \mathrm{~h}$, $18 \mathrm{~h}$ and $24 \mathrm{~h}$ after surgery. The FLACC (Face, Legs, Activity, Cry, Consolability) scale was used to assess the level of pain. The secondary outcome was the frequency of paracetamol/ibuprofen use for pain relief after surgery. Results - Out of the total number of children (100), 70\% were boys (70) and 30\% were girls (30), and their average age was $3.5 \pm 1.9$ years. The average duration of surgical intervention in both groups was 31.6 \pm 4.2 minutes. Significantly fewer children in the experimental group reported pain ( $F L A C C \geq 1)$ at $2 \mathrm{~h}(\mathrm{P}=0.032)$ and $6 \mathrm{~h}(\mathrm{P}=0.001)$ after surgery, compared to the children in the control group $(8$ vs. 17 at $2 \mathrm{~h}$ after surgery; 4 vs. 14 at $6 \mathrm{~h}$ after surgery). Significantly fewer children in the control group reported sensations of pain that required administration of analgesics $6 \mathrm{~h}$ after surgery (FLACC $>3)$ compared to the control group $(\mathrm{P}=0.001)(1$ vs. 14). Overall, significantly fewer children in the experimental group received a paracetamol/ibuprofen mixture for pain relief after surgery, compared to the children in the control group $(\mathrm{P}<0.001)(4$ vs. 50). The average daily amount of acetaminophen in the experimental group was $28 \pm 127 \mathrm{mg} / \mathrm{kg} /$ day and ibuprofen $5.6 \pm 1.8 \mathrm{mg} / \mathrm{kg} / \mathrm{day}$, while in control group, acytaminophen $42.5 \pm 7.7 \mathrm{mg} / \mathrm{kg} /$ day and ibprofen $11.5 \pm 4 \mathrm{mg} / \mathrm{kg} /$ day. The total amount of analgesics was highly statistically significantly lower in the experimental group $(\mathrm{P}<0.001)$. Conclusion - Instillation of levobupivacaine before wound suturing in children who had undergone elective inguinal hernia surgery repair was effective in postoperative pain relief. Children who received levobupivacaine also received less of the paracetamol/ibuprofen mixture to relieve their pain over 24 hours after surgery.
\end{abstract}

Key Words: Children • Surgery • Inguinal Hernia • Pain • Levobupivacaine.

\section{Introduction}

Inguinal hernia is one of the most common conditions in children that requires surgical management (1). A major risk factor for the development of inguinal hernia during childhood is preterm birth (2) and heredity (3). It has been observed that inguinal hernia is more common among boys (4) and tends to appear at an earlier age, compared to girls (1). The repair of inguinal hernia is usually an elective surgical intervention (5). When it becomes 
incarcerated it can be considered an emergency (6). Inguinal hernia surgery is considered a simple intervention, followed by rapid recovery. No complications or pain are expected. Many studies are dedicated to this topic (7-9).

Pain is an important and destructive stressor in surgery, but not the only one. The control of pain during and after surgery significantly reduces perioperative stress. This is perhaps the most important item in postoperative treatment, because treatment of pain is healing in itself, and it allows all the other procedures in postoperative treatment to be performed, which are very often unpleasant and invasive. Stress, including pain in the postoperative period, could contribute to delayed postoperative wound healing (10), cognitive function (11), immune response (12) and development of chronic postsurgical pain (13). All the consequences of untreated pain, in addition to physical, have strong psychological consequences, which could shape the child's attitude towards treatments for life.

The basic postulate in the treatment of a child is non-invasiveness. This is difficult to achieve in paediatric surgery. The child is under a great deal of stress from undergoing tests, the placing of cannulas, separation from parents, the unfamiliar environment, monitors and uniformed people. The goal of modern anaesthesia in outpatient surgery is to provide quick, painless awakening and complete control of postoperative pain. This is best achieved by combining general and local anaesthetics. The trend of maximally reducing opioid use in this type of surgery must also be followed.

Levobupivacaine is a long-acting local anaesthetic which is used in children due to its favourable effects on the cardiovascular and central nervous systems, and very low risk of systemic toxicity $(14,15)$. However, no systematic review of the effectiveness of levobupivacaine for post-surgery pain relief after inguinal hernia repair has been conducted. Perioperative use of levobupivacaine could have a beneficial role in the postoperative recovery from inguinal hernia surgery in terms of pain relief. We hypothesized that children who receive levobupivacaine instillation would have less postoperative pain. We also hypothesized that these children would need less analgesics during the postoperative 24 hours.

The purpose of this study was to compare with a placebo the effectiveness in pain relief of wound infiltration with levobupivacaine in children who underwent inguinal hernia repair. The secondary objective was to examine the frequency of the postoperative analgesics (paracetamol/ibuprofen).

\section{Methods}

\section{Trial Design}

This was a single centre, randomized, placebo controlled study with children who were surgically treated for inguinal hernia.

\section{Participants}

A total of a 100 children who underwent surgery for unilateral inguinal hernia at the "Dr Vukan Čupić" Mother and Child Health Institute, Belgrade, Serbia, were included in this study. The study was conducted from March to December 2013. The inclusion criteria for the study were: age range from 6 months to 7 years, having elective surgery, an American Society of Anesthesiologists (ASA) score of 1 or 2, parental signed informed consent, and oral consent for participation in the study by the surgeon in charge of the operation. They did not have incarcerated hernia, previous surgeries requiring general anaesthesia, allergy to topical anaesthetics, paracetamol, ibuprofen and/or general anaesthetics, opioids or muscle relaxants. The exclusion criteria were: worsening of health status prior to surgery, parental withdrawal from the study, adverse events during surgery or early postoperative period that would otherwise influence the surgical outcomes and postoperative recovery.

\section{Intervention}

All children received midazolam on the paediatric surgery ward before being transported to the operating room, in order to reduce separation anxiety and preparing for anaesthesia. Midazolam was 
administered orally at a dose of $1 \mathrm{mg} / \mathrm{ml}$, mixed with juice for easier consumption. As per the local protocol, children weighing under $10 \mathrm{~kg}$ did not receive midazolam. Children weighing 10-20 $\mathrm{kg}$ received $0.4 \mathrm{mg} / \mathrm{kg}$; children weighing 20-30 $\mathrm{kg}$ received $0.3 \mathrm{mg} / \mathrm{kg}$, children weighing $30-40 \mathrm{~kg}$ received $0.2 \mathrm{mg} / \mathrm{kg}$, and children above $40 \mathrm{~kg}$ received $0.1 \mathrm{mg} / \mathrm{kg}$.

Anaesthesia was performed by using propofol, fentanyl, and cisatracurium as a muscle relaxant in both groups. The usual doses for unilateral herniectomy repair in children for general anaesthesia, were used as follows: fentanyl $3.0-5.0 \mu \mathrm{g} / \mathrm{kg}$, propofol $2.5-3.0 \mathrm{mg} / \mathrm{kg}$, and cisatracurium $0.15 \mathrm{mg} /$ $\mathrm{kg}$. Anaesthesia was maintained with sevoflurane 1 Vol. \%. Children were on artificial ventilation throughout the surgery. No differences were found in the level of fentanyl used for general anaesthesia in the experimental and the control group $(\mathrm{t}=-$ $0.368, \mathrm{P}=0.535)$.

After herniectomy, before suturing of the abdominal fascia, $0.5 \%$ levobupivacaine $0.5 \mathrm{mg} / \mathrm{kg}$ $(0.1 \mathrm{ml} / \mathrm{kg})$ was instilled into the wound in the experimental group. That same amount of $0.9 \%$ saline was given to the children in the control group. The absorption of the solution took approximately 3 minutes, after which the wound was sutured. All the children received $20 \mathrm{mg} / \mathrm{kg}$ of paracetamol as a rectal suppository, before coming round.

After coming round from general anaesthesia, the children were observed until their vital functions were stable. After this, the children were returned to the ward. An anaesthetist, who was blinded to the randomization of the children, monitored their hemodynamic and respiratory stability and reported adverse events or complications. The mixture of paracetamol $10 \mathrm{mg} / \mathrm{kg}$ and ibuprofen $5 \mathrm{mg} / \mathrm{kg}$ as an oral solution was used as the postoperative analgesic. The dosage and timing of postoperative analgesics were reported in a spreadsheet within the medical records.

\section{Outcomes}

The primary outcome was the level of post-operative pain. The secondary outcome was the frequency of paracetamol/ibuprofen use for pain relief after surgery. To quantify the level of postoperative pain, we used the Face, Legs, Activity, Cry, Consolability (FLACC) scale (17). This observational scale is used for assessment of pain in children aged 6 months to 7 years, because many in this age group are not able to properly describe the amount of pain verbally. All 5 aspects of behaviour are graded on a 3 -point scale, from 0 to 2 , where 0 suggests that the corresponding aspect does not show any particular behavioural expression/movement, and value 2 suggests intense behavioural expression/ movement. Scores for all 5 aspects are summed in a total FLACC score. The score ranges from 0 to 10 , with higher values suggesting more intense pain. A FLACC score above 3 is indicative of administration of analgesics for pain relief. A FLACC score above 7 is indicative of opioid analgesics use. The assessment of the level of pain using the FLACC scale was performed by an experienced observer.

A spreadsheet with scores for pain was available for each of the children. On the back of the FLACC scale record, the observer recorded a description and the timing of adverse events. Nausea, vomiting, fever, delayed urination, constipation, allergic reactions to medication and changes in tissue around the wound were listed as adverse events. An additional field was left blank in case other adverse events occurred.

The level of pain was assessed immediately after surgery, i.e. after coming round from general anaesthesia. The next assessment was performed $2 \mathrm{~h}$ after the surgery. After this period, the children were allowed to eat. Also after this period the children were allowed to receive post-operative analgesics, if necessary. The assessment of the FLACC score was performed after they came round from general anaesthesia (t0) and at $2 \mathrm{~h}, 6 \mathrm{~h}, 12 \mathrm{~h}, 18 \mathrm{~h}$ and $24 \mathrm{~h}$ after surgery. The FLACC score was also assessed when the observers were told by the parents or the children, or assessed by themselves that the child needed analgesics. The observer was blinded to levobupivacaine/placebo allocation.

If the observer deemed it necessary to give an analgesic, he was obliged to evaluate the effectiveness of the analgesia 15 minutes after administra- 
tion using the FLACC score. All evaluations and administration of drugs were recorded in the lists provided for that purpose.

\section{Sample Size}

The study sample was calculated on the basis of the number of children treated for inguinal hernia needed to observe a difference in intervention research ( 40 subjects), a type I error (alpha) of 0.05 and a power of $80 \%(16)$. The calculated sample size per study group was 49 , i.e. 49 children were required for the experimental group and 49 children were required for the control group.

\section{Randomization}

The randomization procedure was performed before the surgical procedure to classify the participants into two groups: the experimental and control groups. Simple randomization was applied by flipping a coin before each intervention, whereby "tails" indicated allocation to the experimental group and "heads" indicated allocation to the control group. This approach is considered to be the easiest randomization method in clinical studies (18). An experienced anaesthesiologist-investigator performed randomization as well as wound infiltration.

\section{Blinding}

All participants remained blind to levobupivacaine/ placebo allocation. The assessment of pain was performed by experienced nurses from the intensive care unit, who were also blinded to the allocation of levobupivacaine/placebo. The anaesthesiologistinvestigator and the surgeon in charge of the operation were not blinded. However, they were not involved in the primary outcome assessment. The anaesthesiologist-investigator was the only person who kept a record of levobupivacaine/placebo allocation.

\section{Ethics Statement}

Approval for the study was obtained from the Ethics Committee of the "Dr Vukan Čupić" Mother And Child Health Institute, Belgrade, Serbia (approval no. 4042/2, issued on June 11, 2011). Clinical trial number: This trial was registered at www.clinicaltrails.gov under the identifier NCT04869046.

\section{Statistical Analyses}

Data were analysed using the Statistical Package for Social Sciences (SPSS), version 20. Count and percentages were used to describe the demographic and clinical characteristics of the participants. The normality of distribution was verified using the Kolmogorov Smirnov test. Differences in normally distributed continuous variables were evaluated using the t-test. The Mann-Whitney test was used to evaluate differences between continuous variables that were not normally distributed. Differences in categorical variables were assessed using the Chi square test (for variables with 2 categories and $\geq 5$ observations per cell), the Chi square linear-bylinear association (for variables with more than 2 categories) and Fischer's exact test (for categorical variables with less than 5 observations per cell). We used a linear regression equation to assess the FLACC score trends over a period of 24 hours. The value of the F-test indicated the level of probability of the linear regression coefficient. The linear trend of the FLACC scores over time was assessed in the experimental and the control groups, and according to value of the FLACC score $(\geq 1$ for occurrence of any pain and $>3$ for the level of pain indicative of analgesic administration). The probability level of $\mathrm{P}<0.05$ was considered statistically significant.

\section{Results}

\section{Characteristics of the Participants}

A total of 100 children who met the criteria were included in this study. There were no children who withdrew from the study or who were lost-to-follow up (Fig. 1). Therefore, all the participants who enrolled in the study also completed the follow-up. Fifty participants received levobupivacaine and fifty received a placebo. Of these, $70(70 \%)$ were boys and $30(30.0 \%)$ were girls. 


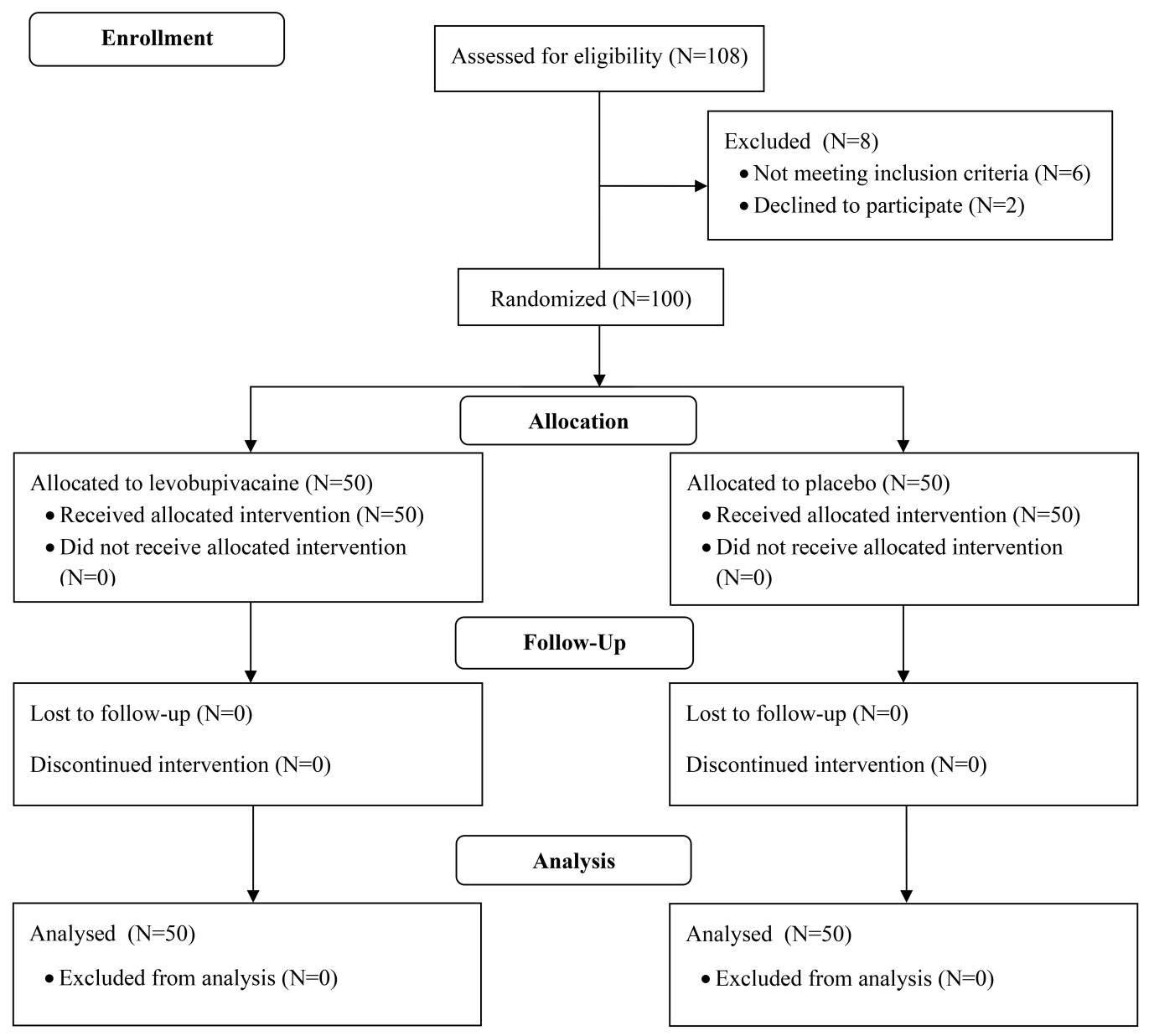

Fig. 1. Diagram of the enrolment and allocation of levobupivacaine and placebo.

\section{Table 1. Socio-Demographic and Clinical Characteristics} of Children

\begin{tabular}{|c|c|c|c|}
\hline \multirow[t]{2}{*}{ Variable } & $\begin{array}{l}\text { Experimental } \\
\text { group } \mathrm{N}=50\end{array}$ & $\begin{array}{l}\text { Control group } \\
\mathrm{N}=50\end{array}$ & \multirow[t]{2}{*}{ P-value* } \\
\hline & $\mathrm{N}(\%)$ & $\mathrm{N}(\%)$ & \\
\hline \multicolumn{4}{|l|}{ Gender } \\
\hline Male & $32(64.0)$ & $38(76.0)$ & \multirow{2}{*}{0.138} \\
\hline Female & $18(36.0)$ & $12(24.0)$ & \\
\hline Age $($ mean $\pm \mathrm{SD})$ & $3.4 \pm 1.9$ & $3.5 \pm 1.9$ & 0.674 \\
\hline $\mathrm{BMI}($ mean $\pm \mathrm{SD})$ & $16.0 \pm 6.4$ & $16.6 \pm 5.7$ & 0.153 \\
\hline \multicolumn{4}{|c|}{ Localization of hernia } \\
\hline Right & $26(52.0)$ & $25(50.0)$ & \multirow{2}{*}{0.600} \\
\hline Left & $24(48.0)$ & $25(50.0)$ & \\
\hline
\end{tabular}

"For difference between the groups; Chi square test was used to assess the difference between genders and localization of hernia; MannWhitney test was used to assess the difference in age and BMI between groups; $\mathrm{SD}=$ Standard deviation; $\mathrm{BMI}=$ Body mass index.
Their basic demographic and clinical characteristics according to the groups are presented in Table 1. No difference in demographic and clinical characteristics was found between the groups (Table 1). The average duration of surgical intervention was $31.6 \pm 4.2$ minutes. The duration of surgery was shorter in girls compared to boys $(25.8 \pm 3.3$ vs. $34.0 \pm 5.1$ minutes, $\mathrm{P}=0.037$ ).

\section{Primary Outcomes}

After surgery, at baseline pain assessment (t0), none of the 50 children in the experimental group or the 48 children in the control group reported any pain (FLACC $\geq 1)(\mathrm{P}=0.495)$. The dynamics of reporting pain and pain that required administration of analgesics according to the groups are presented in 

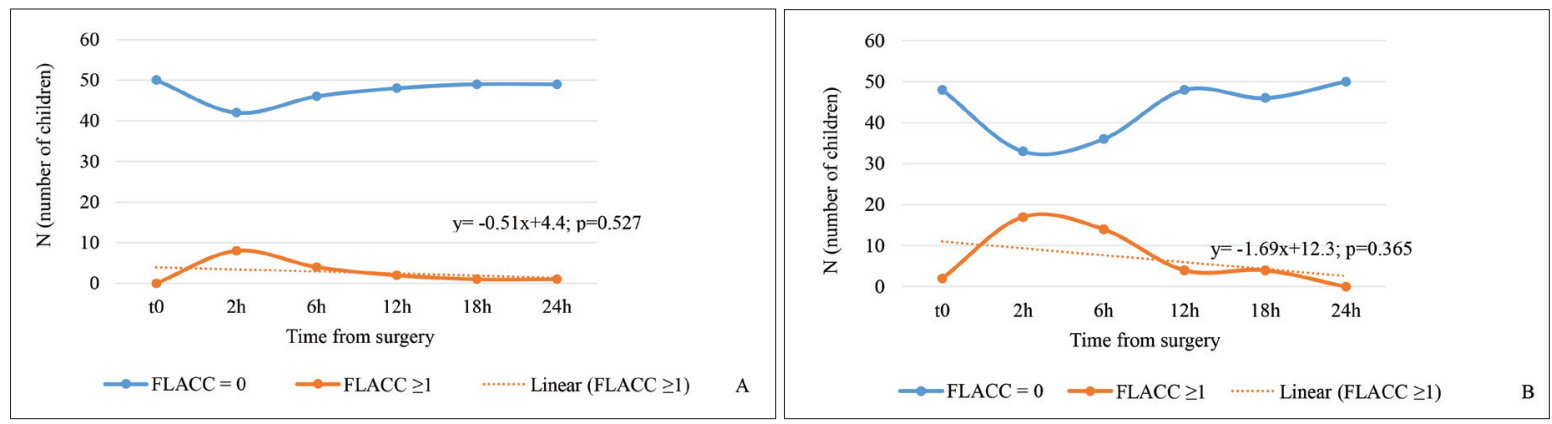

Fig 2. Presence of any level of pain, as measured by the Face, Legs, Activity, Cry, Consolability (FLACC) scale in children after inguinal hernia surgery over time (FLACC score $\geq 1$ is indicative of any pain): $A$ ) in the experimental group; $B$ ) in the control group.

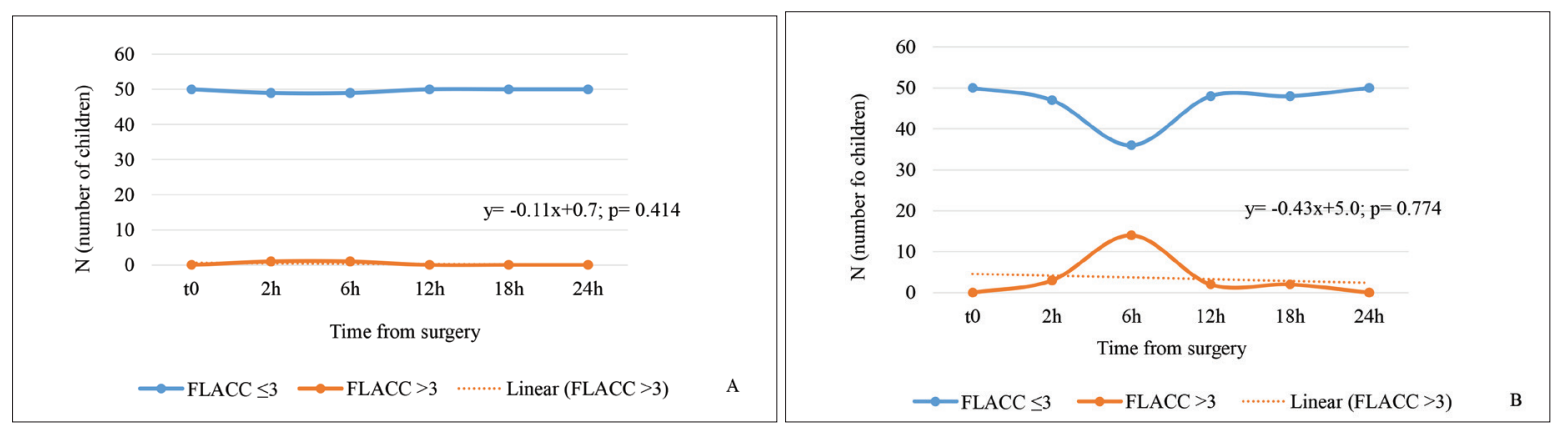

Fig. 3. Presence of pain that required administration of analgesics as measured by the Scores on the Face, Legs, Activity, Cry, Consolability (FLACC) scale over time after inguinal hernia surgery (FLACC score $>3$ is indicative of analgesic administration for pain relief): A) children in the experimental group; B) children in the control group.

Fig. 2 and 3, respectively. Significantly fewer children in the experimental group reported pain at $2 \mathrm{~h}$ $(\mathrm{P}=0.032)$ (8 vs. 17$)$ and $6 \mathrm{~h}(\mathrm{P}=0.001)$ (4 vs. 14$)$ after surgery compared with the control group. This difference was not observed at $12 \mathrm{~h}(\mathrm{P}=0.677), 18 \mathrm{~h}$ $(\mathrm{P}=0.362)$ and $24 \mathrm{~h}(\mathrm{P}=1.000)$ after surgery. Significantly fewer children in the control group reported pain that required administration of analgesics $6 \mathrm{~h}$ after surgery (FLACC >3) compared to the control group $(\mathrm{P}=0.001)$. The level of pain that required administration of analgesics did not differ between the groups at $2 \mathrm{~h}(\mathrm{P}=0.309), 12 \mathrm{~h}(\mathrm{P}=0.495), 18 \mathrm{~h}$ $(\mathrm{P}=0.495)$ and $24 \mathrm{~h}(\mathrm{P}=1.000)$ after surgery.

\section{Secondary Outcomes}

In the experimental group, the average time to the first administration of the analgesics mixture after surgery was 6.5 hours (range 2.5-10 hours). In this group the highest FLACC score indicative of analgesic administration was 5 out of 10 . In the control group, all children received at least one dose of analgesics mixture. In this group the average time to first administration of analgesics after surgery was 5.3 hours (range 1.2-10.8 hours). The highest FLACC score indicative of analgesics administration in this group of children was 7 out of 10. Significantly fewer children in the experimental group received post-surgery analgesics compared with the children in the control group $(\mathrm{P}<0.001)$ (Table 2$)$.

In terms of dosages of post-surgery analgesics, the children in the experimental group received lower doses of acetaminophen compared to the children in the control group $(28.8 \pm 12.7 \mathrm{mg} / \mathrm{kg} /$ day vs. $42.5 \pm 7.7 \mathrm{mg} / \mathrm{kg} /$ day; $\mathrm{P}=0.005)$. Also, the children in the experimental group received lower doses of ibuprofen compared with the children in the control group $(5.6 \pm 1.8 \mathrm{mg} / \mathrm{kg} /$ day vs. $11.5 \pm 4.0$ 
Table 2. Frequency of Analgesic Administration due to Post-operative Pain according to Groups

\begin{tabular}{llll}
\hline $\begin{array}{l}\text { Number of } \\
\text { doses }\end{array}$ & Experimental group & Control group & P-value* \\
\hline 0 & 46 & 0 \\
\hline 1 & 4 & 50 & 0.001 \\
\hline 2 & 1 & 45 & \\
\hline 3 & 0 & 25 & \\
\hline
\end{tabular}

* For difference between the groups, Chi square linear-by-linear association was used to assess the difference between the groups.

$\mathrm{mg} / \mathrm{kg} / \mathrm{day} ; \mathrm{P}=0.006)$. If we compare the individual daily doses given during the observation, with the maximum allowed doses for the age, we observe that in both groups the children received significantly lower doses of acetaminophen and ibuprofen in the mixture than the amount recommended for each drug individually. No adverse events were reported.

\section{Discussion}

This study found that the children who received intraoperative instillation of levobupivacaine during inguinal hernia repair had lower levels of overall pain at $2 \mathrm{~h}$ and $6 \mathrm{~h}$ after surgery compared to the children who did not receive levobupivacaine. Also, the children who received levobupivacaine had lower levels of pain that required administration of analgesics for pain relief at $6 \mathrm{~h}$ following surgery compared to the children who did not receive levobupivacaine. Overall, children who received levobupivacaine required postoperative analgesics for pain relief less often compared to children who did not receive levobupivacaine.

\section{Interpretation of the Study Results}

Possible explanations for the effectiveness of intraoperative levobupivacaine instillation for postoperative pain may be the following: 1) a high anaesthetic concentration was administered in the close proximity of the nerves which innervate the hernia sac; 2) the intrinsic vasoactivity of levobupivacaine allows for longer retention of the drug at the instillation site (this means that resorption is lower and adverse systemic effects are less likely); 3 ) in general anaesthesia the level of drug clearance is lower due to the decreased hepatic blood flow; 4) the perioperative doses of fentanyl were sufficient to relieve the perioperative pain, therefore, levopubivacaine only affected the post-operative pain sensation; 5) the concomitant use of acetaminophen administered rectally elevated the pain threshold for post-operative pain, and thus had a co-analgesic role.

Local anaesthetics are effective in the reverse blocking of impulses along the axons and other excitatory membranes which use $\mathrm{Na}^{+}$channels as the primary site of action. Clinically, local anaesthetics block the pain sensation at the site of application. Unlike systemic drugs, local anaesthetics compete between the perineural uptake and transition to the systemic circulation. The direct measurement of the intraneural concentration of local anaesthetics showed that less than $2-3 \%$ of the injected dose is found inside the nerve (within 30 minutes of administration), and more than $90 \%$ enter the systemic circulation (19).

The goal of perioperative analgesia is to minimize the pain sensations from the surgical wound and to enable the best possible control of pain in the postoperative period. Clinically, this means that the child should come round from the general anaesthesia without a sensation of pain. The primary concern for children who have undergone general anaesthesia and opioid analgesia is to monitor drowsiness, cardiovascular and ventilatory depression, and nausea and vomiting. In the period after surgery, the purpose of combining different analgesics is to optimize the post-operative outcomes: early discharge from the hospital, improve the quality of life and reduce morbidity.

The pain assessment was set at 6h-intervals, because analgesics were administered at regular time intervals. The only exception was the pain assessment $2 \mathrm{~h}$ after surgery. This is because, according to the protocol, children are allowed food intake after $2 \mathrm{~h}$, given the preoperative fasting. The children were discharged after having spent $24 \mathrm{~h}$ on 
the ward. Because of the direct application of the anaesthetic, the possibility of analgesic failure was minimized. Also, thanks to the direct application of the local anaesthetic, an almost 5 times lower dose than the maximum allowed dose of analgesics had an excellent analgesic effect. The expected adverse reactions were inadequate analgesia, local reaction to the anaesthetic, and delayed urine derivation in the post-operative period (albeit very rare in children). However, adverse reactions were not reported, which is in line with previous studies (7, $9,20,21)$.

\section{Limitations of the Study}

This study did not include long-term follow up of the participants. We only assessed the level of pain and the frequency of analgesic administration over a 24-hour period. The core outcome set for analysis of acute pain in the children was not applied. This approach would warrant a longer follow-up period. Ideally, a prospective cohort study could provide more detailed insights into the dynamics of the post-operative period.

Research on analgesia in children is challenging because it requires a delicate balance between scientific, ethical and practical approaches (22). Perception of pain and response to analgesics is exceptionally variable among children. Although placebocontrolled trials are the preferred methodology for the evaluation of drug effectiveness and associated adverse effects, placebo-controlled studies in children pose serious ethical and practical challenges (23). Because children do not have decision-making capacity, exposure to minimum risk needs to be justified by the perceived and expected benefits for each child individually (24). In general, the child should not be exposed to pain greater than the minimum when an effective treatment for pain is available in a given situation.

Research in the paediatric population is also challenged by three aspects that are intertwined and more pronounced at an earlier age: a) there is still no agreement on the design of studies exploring modalities of analgesia and quantification of the analgesic effect; b) pain in young children is always evaluated by a surrogate (either a parent or trained observer), so the basic postulate of pain assessment - subjective experience, can never be implemented c) there is limited prediction of efficacy and risk due to developmental differences between children at different ages (25).

\section{Generalizability}

The results of this study could be generalized to paediatric patients who are scheduled to undergo elective surgery for inguinal hernia, as the best practices for randomized controlled trials were followed. However, there are some other aspects that need to be discussed. Previous studies have reported the presence of post-operative pain at large, despite the availability of various effective anaesthetic techniques and protocols (26). In fact, most physicians believe that the issue in paediatric analgesia is not a lack of effective analgesia for postoperative pain relief, but a lack of accurate assessment of pain and adequate organization of pain treatment (27). The realistic goals of pain treatment in children are to: recognize the pain, anticipate and prevent pain if possible, reduce moderate and severe pain, avoid serious adverse effects, shorten hospital stay, and prevent the development of chronic pain syndromes. In ideal conditions, successful treatment of pain in hospitalized children would be based on an individually-tailored analgesic plan for each child. Such a plan would include the specificities of age and the health condition, as well as the specificities of the surgical procedure and the associated risks, along with use of the available analgesics and nonpharmacological procedures for pain relief.

\section{Conclusion}

Instillation of levobupivacaine before wound suturing in children undergoing inguinal hernia surgery repair was effective in post-operative pain relief. Children who received levobupivacaine also received less paracetamol/ibuprofen to relieve their pain over 24 hours after surgery. Thanks to the 
direct application of a local anaesthetic, an almost 5 times lower dose than the maximum allowed dose of analgesics had good analgesic effect and meant a lower risk of side effects. Intraoperative instillation of levobupivacaine could be used as routine analgesia for postoperative pain relief.

Acknowledgement: The authors are grateful to all the parents of children who participated in this study. The authors would also like to thank all the staff from the paediatric surgery department, especially the Anaesthesia Service of the "Dr Vukan Čupić” Mother And Child Health Institute, Belgrade, Serbia for their support.

Authors' Contributions: Conception and design: MŠ, TG, SV, AM, IK; Acquisition, analysis and interpretation of data: MŠ, TG, SV, AM, IK; Drafting the article: MŠ, TG; Revising it critically for important intellectual content:, SV, AM, IK; Approved final version of the manuscript: MŠ, TG, SV, AM, IK.

Clinical Trial Number: This trial was registered at www.clinicaltrails.gov under the identifier NCT04869046.

Conflict of Interest: The authors declare that they have no conflict of interest.

\section{References}

1. Chang SJ, Chen JY, Hsu CK, Chuang FC, Yang SS. The incidence of inguinal hernia and associated risk factors of incarceration in pediatric inguinal hernia: a nation-wide longitudinal population-based study. Hernia. 2016;20(4):559-63.

2. Fu YW, Pan ML, Hsu YJ, Chin TW. A nationwide survey of incidence rates and risk factors of inguinal hernia in preterm children. Pediatr Surg Int. 2018;34(1):91-5.

3. Zöller B, Ji J, Sundquist J, Sundquist K. Shared and nonshared familial susceptibility to surgically treated inguinal hernia, femoral hernia, incisional hernia, epigastric hernia, and umbilical hernia. J Am Coll Surg. 2013;217(2):28999.

4. Chen $\mathrm{YH}$, Wei CH, Wang KK. Children With Inguinal Hernia Repairs: Age and Gender Characteristics. Glob Pediatr Health. 2018;5:2333794X18816909.

5. Hirabayashi T, Ueno S, Hirakawa H, Tei E, Mori M. Surgical Treatment of Inguinal Hernia with Prolapsed Ovary in Young Girls: Emergency Surgery or Elective Surgery. Tokai J Exp Clin Med. 2017;42(2):89-95.

6. Verhelst J, de Goede B, van Kempen BJ, Langeveld HR, Poley MJ, Kazemier G et al. Emergency repair of inguinal hernia in the premature infant is associated with high direct medical costs. Hernia. 2016;20(4):571-7.

7. Sharma J, Gupta R, Kumari A, Mahajan L, Singh J. A Comparative Study of $0.25 \%$ Levobupivacaine, $0.25 \%$ Ropivacaine, and $0.25 \%$ Bupivacaine in Paediatric Single Shot Caudal Block. Anesthesiol Res Pract. 2018;2018:1486261.

8. Yao Y, Yu C, Zhang X, Guo Y, Zheng X. Caudal and intravenous dexmedetomidine similarly prolong the duration of caudal analgesia in children: A randomized controlled trial. Paediatr Anaesth. 2018;28(10):888-96.

9. Marjanovic V, Budic I, Stevic M, Simic D. A Comparison of Three Different Volumes of Levobupivacaine for Caudal Block in Children Undergoing Orchidopexy and Inguinal Hernia Repair. Med Princ Pract. 2017;26(4):331-6.

10. Walker SM. Pain in children: recent advances and ongoing challenges. Br J Anaesth. 2008;101(1):101-10.

11. Safavynia SA, Goldstein PA. The Role of Neuroinflammation in Postoperative Cognitive Dysfunction: Moving From Hypothesis to Treatment. Front Psychiatry. 2019;9:752.

12. Haroutounian S. Postoperative opioids, endocrine changes, and immunosuppression. Pain Rep. 2018;3(2):e640.

13. Williams G, Howard RF, Liossi C. Persistent postsurgical pain in children and young people: prediction, prevention, and management. Pain Rep. 2017;2(5):e616.

14. Foster RH, Markham A. Levobupivacaine: a review of its pharmacology and use as a local anaesthetic. Drugs. 2000;59(3):551-79.

15. Sola C, Menacé C, Bringuier S, Saour AC, Raux O, Mathieu $\mathrm{O}$ et al. Transversus Abdominal Plane Block in Children: Efficacy and Safety: A Randomized Clinical Study and Pharmacokinetic Profile. Anesth Analg. 2019;128(6):1234-41.

16. Browner WS, Newman TB, Hulley SB. Estimating Sample Size and Power: Applications and Examples. In: Hulley SB, Cummings SR, Browner WS, Grady DC, Newman TB (Eds). Designing Clinical Research. 4th Ed. Lippincot Williams \& Willkins; 2013; pp.55-82.

17. Voepel-Lewis T, Zanotti J, Dammeyer JA, Merkel S. Reliability and validity of the face, legs, activity, cry, consolability behavioral tool in assessing acute pain in critically ill patients. Am J Crit Care. 2010;19(1):55-61.

18. Suresh KP. An overview of randomization techniques: An unbiased assessment of outcome in clinical research J Hum Reprod Sci. 2011;4(1):8-11.

19. Gupta A. Wound infiltration with local anaesthetics in ambulatory surgery. Curr Opin Anaesthesiol. 2010;23(6):705. 
20. Dogra N, Dadheech R, Dhaka M, Gupta A. A study to compare caudal levobupivacaine, tramadol and a combination of both in paediatric inguinal hernia surgeries. Indian J Anaesth. 2018;62(5):359-65.

21. Cicekci F, Sizer C, Atici SS, Arican S, Karaibrahimoglu A, Kara I. Comparison of the Perioperative and Postoperative Effects of Levobupivacaine and of Levobupivacaine + Adrenaline in Pediatric Tonsillectomy: A Double-Blind Randomized Study. Pain Res Manag. 2017;2017:8431823.

22. Rawal N. Current issues in postoperative pain management. Eur J Anaesthesiol. 2016;33(3):160-71.

23. Ferland CE, Vega E, Ingelmo PM. Acute pain management in children: challenges and recent improvements. Curr Opin Anaesthesiol. 2018;31(3):327-32.

24. Sanders RD, Ma D, Brooks P, Maze M. Balancing paediatric anaesthesia: preclinical insights into analgesia, hyp- nosis, neuroprotection, and neurotoxicity. $\mathrm{Br} \mathrm{J}$ Anaesth. 2008;101(5):597-609.

25. Matsota P, Papageorgiou-Brousta M, Kostopanagiotou G. Wound infiltration with levobupivacaine: an alternative method of postoperative pain relief after inguinal hernia repair in children. Eur J Pediatr Surg. 2007;17(4):270-4.

26. Zielinska M, Bartkowska-Sniatkowska A, Becke K, Höhne C, Najafi N, Schaffrath E et al. Safe pediatric procedural sedation and analgesia by anesthesiologists for elective procedures: A clinical practice statement from the European Society for Paediatric Anaesthesiology. Paediatr Anaesth. 2019;29(6):583-90.

27. Ross A, Young J, Hedin R, Aran G, Demand A, Stafford A et al. A systematic review of outcomes in postoperative pain studies in paediatric and adolescent patients: towards development of a core outcome set. Anaesthesia. 2018;73(3):375-83. 